\title{
Lesson of the month 1: Prolonged QT syndrome due to donepezil: a reversible cause of falls?
}

\author{
Authors: Emily G Jackson ${ }^{A}$ and Sarah Stowe ${ }^{B}$
}

\begin{abstract}
Prolonged QT syndrome precipitates cardiac arrhythmias such as torsades de pointes (TdP) resulting in cardiogenic syncope or sudden death. We report a case of prolonged QT syndrome caused by donepezil which resulted in a fall and hip fracture. In this case female sex, advanced age and diuretic use may have increased the risk of recurrent syncope and potential underlying TdP. Cessation of donepezil resulted in normalisation of the QT interval. This case highlights a lesser known side effect of this dementia drug. It also reminds us of the importance of taking a thorough drug history while considering potential drug toxicity/interactions as part of the comprehensive geriatric assessment.
\end{abstract}

KEYWORDS: Donepezil, falls, QT interval, torsades de pointes, syncope

\section{Case presentation}

An 83 year old Caucasian female presented to the emergency department with hip pain after an unwitnessed syncopal episode. She denied any prodromal symptoms and there was no post-ictal phase, tongue biting or incontinence. A fractured neck of the femur was diagnosed and she was transferred to the orthopaedic trauma ward. Her past medical history included early Alzheimer's dementia, hypertension and recurrent falls (although she had not previously sought medical attention for this problem). Her admission medications were bendroflumethiazide $2.5 \mathrm{mg}$, simvastatin $20 \mathrm{mg}$ and donepezil $10 \mathrm{mg}$. She lived alone and was independent with activities of daily living (ADLs).

On admission the patient's electrocardiogram (ECG) demonstrated normal sinus rhythm with a prolonged corrected QT interval (QTc) of $638 \mathrm{~ms}$ (the upper limit of normal for females is $460 \mathrm{~ms}$ ). ${ }^{1}$ Blood tests, including electrolytes, were all within normal limits and there was no postural blood pressure deficit. The patient was reviewed by the orthogeriatrics team for falls and bone health assessment, and medical optimisation prior to surgery. Given her history of syncope without prodromal symptoms, we suspected that this patient had fallen as a result of cardiogenic syncope

Authors: ${ }^{\text {A }}$ core medical trainee, Chesterfield Royal Hospital NHS Foundation Trust, Chesterfield, UK; ${ }^{B}$ consultant geriatrician, Airedale General Hospital, Keighley, UK relating to prolonged QT syndrome. Donepezil was withdrawn as the potentially causative medication. The 12 lead ECG was repeated at days 2 and 10 , and the QTc had returned to normal ( $436 \mathrm{~ms}$ ) by day 10 . Subsequent echocardiogram and $24 \mathrm{~h} \mathrm{ECG}$ were normal. Following surgical intervention for the fracture, and input from the multi-disciplinary team, the patient was transferred to a community rehabilitation facility.

\section{Discussion}

The QT interval is the measurement between the start of the QRS complex and the end of the T wave, and it represents the duration of ventricular repolarisation. ${ }^{1}$ There are multiple factors that affect the duration of the QT interval including sex, heart rate and underlying heart rhythm. In clinical practice it is most relevant to correct QT for heart rate; Bazett's formula $(\mathrm{QTC}=\mathrm{QT} / \sqrt{\mathrm{RR}})$ is most widely used. Drug interactions can lead to a cumulative effect on QT interval although this has an unpredictable effect in causing TdP and is affected by many risk factors. ${ }^{2}$ In this case the only causative medication was donepezil, although female sex, advanced age and use of diuretics were risk factors for TdP with prolonged QT.

Donepezil is a reversible inhibitor of acetylcholinesterase for use in mild to moderate dementia in Alzheimer's disease. It has multiple known cardiac side-effects including dizziness, syncope, bradycardia, atrioventricular block, and sinoatrial block. ${ }^{3}$ In recent years, cases of donepezil-induced QT prolongation and TdP have been reported but this is not widely known and it is not a listed side effect in the British National Formulary. ${ }^{3-7}$ The list of drugs known to cause prolonged QT syndrome is long and frequently growing. The website CredibleMeds.org, published in conjunction with the US Food and Drug Administration, is a regularly updated list of drugs with a risk of QT interval prolongation and cardiac arrhythmias. Indeed, donepezil is listed as a 'known risk of TdP' on this resource. ${ }^{8}$

People living with dementia have an increased risk of falls. This is related to multiple factors including impaired gait, balance and orthostatic hypotension coupled with changes in cognition, attention and behaviour. ${ }^{7}$ Furthermore people living with dementia are more likely to suffer the serious consequences of falls and less likely to recover from their injuries. ${ }^{9}$ A recent Cochrane review of the use of donepezil in Alzheimer's disease has found only modest benefit in terms of cognition and ability to perform ADLs with no improvement in quality of life. Concern was also raised about the rate of adverse effects with donepezil 
which increased with higher doses. ${ }^{10}$ With this in mind, clinicians should consider the cardiac side effects and measure a baseline QT interval prior to commencing therapy.

\section{Key points}

> Donepezil causes QT prolongation but this is not widely known.

$>$ Growing evidence is emerging of the significant potential harms related to donepezil with only modest benefit.

> Clinicians should start this drug with caution after careful consideration of the cardiac side effects and risk of falls.

> Prior to starting donepezil, a 12 lead ECG should be performed to document baseline heart rhythm and QTc and risk factors for $\mathrm{TdP}$ should be considered.

> Patients should be informed of the potential for cardiac arrhythmias and falls when starting donepezil.

\section{Consent}

Consent was obtained to publish the clinical details in this article.

\section{References}

1 Rautaharju PM, Surawicz B, Gettes LS. AHA/ACCF/HRS recommendations for the standardisation and interpretation of the electrocardiogram. Part IV: the ST segment, T and U Waves, and QT interval. J Am Coll Cardiol 2009;53:982-91.
2 Nachimuthu S, Assar MD, Schussler JM. Drug-induced QT interval prolongation: mechanisms and clinical management. Ther Adv Drug Saf 2012;3:241-53.

3 Joint Formulary Committee. British National Formulary. London: BM] Group and Pharmaceutical Press, 2018.

4 Kitt J, Irons R, Al-Obaidi M, Missouris C. A case of donepezil-related torsades de pointes. BMJ Case Rep 2015;2015:bcr2015211900.

5 Gurbuz AS, Ozturk S, Acar E et al. Acquired long QT syndrome and torsades de pointes related to donepezil use in a patient with Alzheimer disease. Egypt Heart J 2016;68:197-9.

6 Tayaka T, Okamoto M, Yodoi K et al. Torsades de pointes with QT prolongation related to donepezil use. J Cardiol 2009;54:507-11.

7 Kim DH, Brown RT, Ding EL, Kiel DP, Berry SD. Dementia medications and risk of falls, syncope, and related adverse events meta-analysis of randomized controlled trials. J Am Geriatr Soc 2011;59:1019-31.

8 QTdrugs List, 2018. https://crediblemeds.org/new-drug-list/ [Accessed 30 August 2018].

9 Allan LM, Ballard CG, Rowan EN, Kenny RA. Incidence and prediction of falls in dementia: a prospective study in older people. PLOS ONE 2009;4:e5521.

10 Birks JS, Harvey RJ. Donepezil for dementia due to Alzheimer's disease. Cochrane Database Syst Rev 2018;6:CD001190.

Address for correspondence: Dr Emily Georgina Jackson, Chesterfield Royal Hospital NHS Foundation Trust, Cardiology, Chesterfield Road, Calow, Chesterfield, S44 5BL, UK.

Email: emilygjackson@doctors.org.uk

\section{NGC

\section{New to systematic reviewing, or need} to improve your critical appraisal skills?

Our intensive 1-day courses will introduce you to the key principles of critical appraisal and systematic reviewing, and equip you with the basic skills to put your knowledge into practice.

\section{$>$ Introduction to critical appraisal}

> Systematic review and meta-analysis in action

Discount available if both courses are booked together.

\section{'Informative and practical'}

Dr Rajeswari Ramaraj

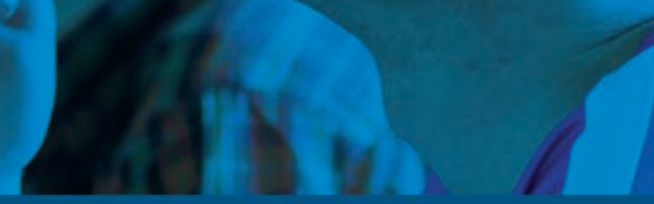

For course dates and more information visit: www.rcplondon.ac.uk/ngc-training-courses 